Eds.) pp 49-56, ESCOM Science Publishers, Leiden, The Netherlands.

Hamoir, G., \& Konosu, S. (1965) Biochem. J. 96, 85-97.

Hendrikson, W. A., \& Karle, J. (1973) J. Biol. Chem. 248, 3327-3334.

James, D. R., Liu, Y.-S., Siemiarczuk, A., Wagner, B. D., \& Ware, W. R. (1988) Proc. SPIE-Int. Soc. Opt. Eng. 909, $90-96$.

Kretsinger, R. H. (1980) CRC Crit. Rev. Biochem. 12, 119-174.

Kretsinger, R. H., Dangelat, D., \& Bryan, R. F. (1971) J. Mol. Biol. 59, 213-214.

Lakowicz, J. R. (1983) Principles of Fluorescence Spectroscopy, Pleumn Press, New York.

Moews, P. C., \& Kretsinger, R. H. (1975) J. Mol. Biol. 91, 201-228.

Motulsky, H. J., \& Ransnas, L. A. (1987) FASEB J. 1, 365-374.
Pechere, J. F., Demaille, J., \& Capony, J. P. (1971) Biochim. Biophys. Acta 236, 391-408.

Pechere, J. F., Capony, J. P., \& Demaille, J. (1973) Syst. Zool. 22, 533-548.

Permyakov, E. A., Yarmolenko, V. V., Emelyanenko, V. I., Burstein, E. A., Closset, J., \& Gerday, Ch. (1980) Eur. J. Biochem. 109, 307-315.

Permyakov, E. A., Ostrovsky, A. V., Burstein, E. A., Pleshnov, P. G., \& Gerday, Ch. (1985) Arch. Biochem. Biophys. 240, 781-792.

Robertson, S. P., Johnson, J. D., \& Potter, J. D. (1981) Biophys. J. 34, 559-569.

Schwarzenbach, G. (1957) Compleximetric Titrations, Interscience, New York.

Szabo, A. G., \& Rayner, D. M. (1980) J. Am. Chem. Soc. $102,554-563$.

Weber, G. (1977) J. Chem. Phys. 66, 4081-4091.

White, H. D. (1988) Biochemistry 27, 3357-3365.

\title{
The Nature of Protein Dipole Moments: Experimental and Calculated Permanent Dipole of $\alpha$-Chymotrypsin
}

\author{
Jan Antosiewicz $z^{\ddagger}$ and Dietmar Porschke* \\ Max-Planck-Institut für biophysikalische Chemie, D-3400 Göttingen, FRG \\ Received January 25, 1989; Revised Manuscript Received July 28, 1989
}

\begin{abstract}
The electric dichroism of $\alpha$-chymotrypsin has been measured in buffers of various $\mathrm{pH}$ values and ion compositions. The stationary dichroism obtained as a function of the electric field strength is not compatible with an induced dipole mechanism and clearly shows that $\alpha$-chymotrypsin is associated with a substantial permanent dipole moment. After correction for the internal directing electric field according to a sphere model, the dipole moment is $1.6 \times 10^{-27} \mathrm{C} \mathrm{m}$ at pH 8.3 (corresponding to $480 \mathrm{D}$ ). This value decreases with decreasing $\mathrm{pH}$ (to $1.2 \times 10^{-27} \mathrm{C} \mathrm{m}$ at $\mathrm{pH} 4.2$ ), but is almost independent of the monovalent salt concentration in the range from 2 to $12 \mathrm{mM}$ and of $\mathrm{Mg}^{2+}$ addition up to $1 \mathrm{mM}$. The assignment of the permanent dipole moment is confirmed by analysis of the dichroism rise curves. The dichroism decay time constants of $(31 \pm 1)$ ns at $2{ }^{\circ} \mathrm{C}$ can be represented by a spherical model with a radius of $25-26 \AA$, which is consistent with the known X-ray structure. The limiting linear dichroism is slightly dependent on the buffer composition and demonstrates subtle variations of the protein structure. As a complement to the experimental results, electric and hydrodynamic parameters of $\alpha$-chymotrypsin have been calculated according to the known X-ray structure. Bead model simulations provide the center of diffusion, which is used to calculate dipole moments according to the equilibrium charge distribution evaluated from standard $\mathrm{p} K$ values. In addition, dipole moments are also calculated according to the Tanford-Kirkwood model, which is modified by a more detailed consideration of solvation energies and evaluated from an equilibrium distribution of protein states generated by Monte Carlo techniques. The static dipole moment obtained by the first procedure is consistent with the experimental results over the whole $\mathrm{pH}$ range investigated, whereas the Monte Carlo results agree with the experimental data from $\mathrm{pH} 8.3$ to 5.7. The fluctuating dipole moment evaluated by the second procedure gives rise to a minor contribution only, which is usually less than $20 \%$ of the static dipole moment. In summary, experimental and theoretical results are in satisfactory agreement and demonstrate the existence of a substantial "true" permanent dipole moment associated with $\alpha$-chymotrypsin.
\end{abstract}

The he assessment of electric properties for biological macromolecules proved to be particularly difficult-mainly due to the fact that these molecules usually bear a large number of charged residues. In most cases these residues attract counterions, which form a counterion atmosphere with a particularly high polarizability and thus cause large contributions to the measured electric properties. Although the polarization

\footnotetext{
¥Present address: Institute of Experimental Physics, Department of Biophysics, University of Warsaw, 02-089 Warsaw, Poland.
}

of counterions should be reflected by an induced dipole moment, the case of DNA double helices (Diekmann et al., 1982; Porschke, 1985) demonstrates that special mechanisms may simulate the existence of a permanent dipole moment. Another problem results from site binding of ligands, which compensate the charges attached to the polymer. Usually the extent of site binding is not exactly known. Because of the large dimensions of macromolecules, the contribution of single charged residues may be important for the overall dipole moment. Furthermore, ligand binding at individual sites may fluctuate, 
and thus individual molecules may have a considerable dipole moment due to these fluctuations (Kirkwood \& Shumaker, 1952). Since the standard approach for the determination of protein dipole moments has been dielectric measurements, which provide the sum of squared moments, fluctuations may lead to large contributions to experimental dipole moments. Due to these difficulties large dipole moments observed for macromolecules are often regarded with great caution or are not taken seriously at all.

Recently, experimental evidence has been accumulated by electrooptical measurements for the existence of large "true" permanent dipole moments for lac and tet repressor proteins. In the case of lac repressor (Porschke, 1987), where the nature of the dipole could be analyzed in detail, the dipole moment is $\sim 4 \times 10^{-27} \mathrm{C} \mathrm{m}$; the tet repressor (Porschke et al., 1988) is associated with a dipole moment of $3.5 \times 10^{-27} \mathrm{C} \mathrm{m}$. Although the methods used in these investigations are particularly safe, it appeared to be useful to do a corresponding analysis on a protein with a known X-ray structure, because in that case the experimental data may be compared with theoretical predictions based on the coordinates of charged residues and hydrodynamic model simulations. Test experiments with various proteins of known crystal structure showed that $\alpha$ chymotrypsin is a good candidate for such investigation owing to its relatively large electric dichroism. Thus, we have used $\alpha$-chymotrypsin as a test object for a detailed comparison of experimental and theoretical results.

\section{Materials and Methods}

$\alpha$-Chymotrypsin (bovine pancreas) was obtained from Serva (Heidelberg, FRG). Protein samples were dialyzed into the following buffers: Al contained $20 \mathrm{mM}$ Tris- $\mathrm{HCl}, \mathrm{pH} 8.3$, $100 \mu \mathrm{M}$ dithioerythritol, and $200 \mu \mathrm{M}$ EDTA; A2 was the same as $\mathrm{A} 1$, but $200 \mu \mathrm{M}$ EDTA was replaced by $200 \mu \mathrm{M} \mathrm{MgCl}$; A3 was the same as A2, but $\mathrm{MgCl}_{2}$ was $1 \mathrm{mM}$; $\mathrm{A} 4$ was the same as $\mathrm{A} 3$ plus $10 \mathrm{mM} \mathrm{NaCl} ; \mathrm{B} 1$ contained $1 \mathrm{mM} \mathrm{NaCl}$, $1 \mathrm{mM}$ sodium cacodylate, $\mathrm{pH} 7.0$, and $200 \mu \mathrm{M}$ EDTA; B2 was the same as B1 plus $5 \mathrm{mM} \mathrm{NaCl}$; $\mathrm{B} 3$ was the same as B1 plus $10 \mathrm{mM} \mathrm{NaCl} ; \mathrm{C}$ contained $10 \mathrm{mM}$ sodium cacodylate, $\mathrm{pH} 5.7$, and $200 \mu \mathrm{M} \mathrm{MgCl}$; D contained $2 \mathrm{mM}$ sodium acetate, $\mathrm{pH} 4.2$, and $200 \mu \mathrm{M} \mathrm{MgCl}_{2}$.

The electric dichroism was measured by a pulse generator (Grünhagen, 1974) and an optical detection system (Porschke, 1980 ) as described. The samples were subjected to field pulses in the range from 5 to $70 \mathrm{kV} / \mathrm{cm}$ in a cell with $10 \mathrm{~mm}$ optical path length and a distance between the Pt electrodes of 6.05 $\mathrm{mm}$. UV radiation damage was avoided by an automatic shutter for short light pulses synchronized to the field pulses. For measurements of the stationary dichroism, both UV transmission and electric field strength were recorded by a Tektronix 7612D transient recorder and evaluated by using graphic routines on a LSI 11/23. (Porschke et al., 1984). Dichroism transients for the determination of time constants were recorded by a Tektronix $7912 \mathrm{AD}$; these data were transmitted to the facilities of the Gesellschaft für wissenschaftliche Datenverarbeitung $\mathrm{mbH}$, Göttingen, and were evaluated by an efficient deconvolution routine (Porschke \& Jung, 1985). Reference curves for deconvolution were obtained by birefringence measurements of water.

\section{RESULTS}

Stationary Dichroism. When solutions of $\alpha$-chymotrypsin are exposed to electric field pulses, the absorbance measured at $280 \mathrm{~nm}$ by light polarized parallel to the field vector increases $\left(\Delta A_{\|}>0\right)$. Since electric field pulses do not induce absorbance changes at the magic angle orientation $\left(\Delta A_{55}=\right.$

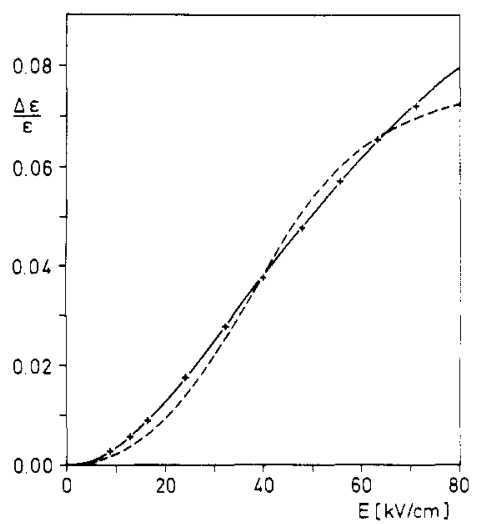

FIGURE 1: Reduced linear dichroism $\Delta \epsilon / \epsilon$ of $\alpha$-chymotrypsin as a function of the electric field strength $E$. The continuous line represents a least-squares fit of the data according to a permanent dipole mechanism with $\mu_{\mathrm{p}}=2.3 \times 10^{-27} \mathrm{C} \mathrm{m}$ and $(\Delta \epsilon / \epsilon)_{\infty}=0.164$. The dashed line represents a least-squares fit according to an induced dipole mechanism (buffer $\mathrm{C}, 2^{\circ} \mathrm{C}$ ).

$0)$ and the absorbance changes $\Delta A_{\perp}$ observed at perpendicular orientation of the polarization plane with respect to the field vector are consistent with $2 \Delta A_{\perp}=-\Delta A_{\sharp}$, obviously the absorbance changes reflect field-induced alignment of $\alpha$-chymotrypsin molecules. The absorbance changes arrive at stationary levels within pulse lengths of about a microsecond. The stationary absorbance changes are used to calculate the reduced linear dichroism according to

$$
\Delta \epsilon / \epsilon=\left(\Delta A_{\|}-\Delta A_{\perp}\right) / A
$$

where $A$ is the isotropic absorbance. The increase of the dichroism $\Delta \epsilon / \epsilon$ with the electric field strength $E$ can be fitted by a permanent dipole mechanism at high accuracy (cf. Figure 1). For least-squares fitting we have used the standard equation (Fredericq \& Houssier, 1973)

$$
\frac{\Delta \epsilon}{\epsilon}=\left[1-\left(\operatorname{coth} \beta-\frac{1}{\beta}\right) \frac{3}{\beta}\right] \frac{\Delta \epsilon_{\infty}}{\epsilon}
$$

with $\beta=\mu_{\mathrm{p}} E / k T . \Delta \epsilon_{\infty} / \epsilon$ is the linear dichroism in the limit of complete alignment, $k T$ thermal energy, and $\mu_{\mathrm{p}}$ permanent dipole moment.

As shown in Figure 1 the experimental data are not consistent with an induced dipole mechanism. The two orientation mechanisms can be clearly distinguished owing to their different increase of the optical anisotropy with the electric field strength. The sum of squared residuals found for the fit according to the induced dipole model was higher by a factor of $\sim 100$ than the corresponding fit according to the permanent dipole model.

The permanent dipole moment evaluated according to eq 2 is $2.3 \times 10^{-27} \mathrm{C} \mathrm{m}$. This value has to be corrected for the difference between the external, applied electric field strength $E_{\mathrm{e}}$ and the internal directing field strength $E_{\mathrm{i}}$. Since $\alpha$-chymotrypsin may be regarded as spherical to a reasonable approximation, the correction factor (Bottcher, 1973) may be calculated according to

$$
E_{\mathrm{i}}=[3 \epsilon /(2 \epsilon+1)] E_{\mathrm{e}}
$$

where $\epsilon$ is the dielectric constant of the solvent. After correction according to this simple model, the dipole moment is $1.5 \times 10^{-27} \mathrm{C} \mathrm{m}$, which corresponds to $460 \mathrm{D}$ (buffer C, 20 $\left.{ }^{\circ} \mathrm{C}\right)$.

The procedure described above has been used to determine the dipole moment under various conditions. As shown by the compilation of results in Table I the dipole moment of $\alpha$ chymotrypsin decreases with decreasing $\mathrm{pH}$. Variations of the 


\begin{tabular}{|c|c|c|c|c|c|c|c|c|}
\hline \multirow[b]{2}{*}{$\mathrm{pH}$} & \multirow[b]{2}{*}{ temp $\left({ }^{\circ} \mathrm{C}\right)$} & \multirow[b]{2}{*}{ buffer } & \multirow{2}{*}{$\begin{array}{l}\text { concn of mono- } \\
\text { valent ions (mM) }\end{array}$} & \multirow{2}{*}{$\begin{array}{l}{\left[\mathrm{Mg}^{2+}\right]} \\
(\mathrm{mM})\end{array}$} & \multicolumn{2}{|c|}{$\mu_{\mathrm{p}}\left(10^{-27} \mathrm{C} \mathrm{m}\right)$} & \multirow[b]{2}{*}{$\Delta \epsilon_{\infty} / \epsilon$} & \multirow[b]{2}{*}{$\tau(\mathrm{ns})$} \\
\hline & & & & & $\mathrm{a}$ & $\mathrm{b}$ & & \\
\hline 8.3 & 20 & Al & 9 & & 2.4 & 1.6 & 0.04 & \\
\hline 8.3 & 20 & A2 & 9 & 0.2 & 2.3 & 1.5 & 0.10 & 29.0 \\
\hline 8.3 & 20 & A3 & 9 & 1.0 & 2.3 & 1.5 & 0.08 & \\
\hline 8.3 & 20 & A4 & 19 & 1.0 & 2.5 & 1.7 & 0.05 & \\
\hline 8.3 & 2 & A2 & 9 & 0.2 & 2.6 & 1.7 & 0.11 & \\
\hline 7,0 & 20 & B1 & 2.4 & & 2.1 & 1.4 & 0.11 & 31.4 \\
\hline 7.0 & 20 & B2 & 7.4 & & 2.1 & 1.4 & 0.13 & \\
\hline 7.0 & 20 & B3 & 12.4 & & 2.1 & 1.4 & 0.13 & \\
\hline 5.7 & 20 & C & 10 & 0.2 & 2.3 & 1.5 & 0.15 & \\
\hline 5.7 & 2 & $\mathrm{C}$ & 10 & 0.2 & 2.3 & 1.5 & 0.17 & 31.4 \\
\hline 4.2 & 2 & D & 2 & 0.2 & 1.8 & 1.2 & 0.16 & 29.7 \\
\hline
\end{tabular}

$a_{\mu_{\mathrm{p}}}$ is the permanent dipole moment; the values given in the column a are not corrected, and those in column b are corrected according to eq 3 (cf. text). $\Delta \epsilon_{\infty} / \epsilon$ is the limit value of the reduced linear dichroism (estimated accuracy $\pm 10 \%$ ). $\tau$ is the dichroism decay time constant (estimated accuracy $\pm 1.5 \mathrm{~ns})$.

ion composition do not affect the magnitude of the dipole moment within the range $2-15 \mathrm{mM}$ monovalent salt and $0-1$ $\mathrm{mM} \mathrm{Mg}{ }^{2+}$.

The limit dichroism $\Delta \epsilon_{\infty} / \epsilon$ is quite sensitive to variations of the ion composition (cf. Table I). Changes of this parameter indicate shifts of positions of aromatic amino acid residues relative to the axis of $\alpha$-chymotrypsin orientation in the electric field. Thus, slight variations in the ion composition induce some changes of the protein structure. Since the dipole moment is not affected, these changes appear to be relatively small.

Rotation Time Constants. Since the time constants of dichroism rise and decay observed for $\alpha$-chymotrypsin are at the limit of time resolution of our experimental technique, these time constants have been determined at $2^{\circ} \mathrm{C}$, where the increased viscosity of water decreases the rate of rotational diffusion. The decay curves, which have been fitted by a deconvolution technique, can be represented by single exponentials at high accuracy. The decay time constants do not show any detectable dependence on the field strength of the pulses applied for orientation. As shown in Table I the decay time constants are also not dependent on $\mathrm{pH}$ within experimental accuracy.

By use of the friction coefficient for spherical objects (Cantor \& Schimmel, 1980), the decay time constants $\tau_{\mathrm{d}}$ may be used to calculate the effective hydrated volume $V_{\mathrm{h}}$ of $\alpha$ chymotrypsin according to

$$
\tau_{d}=V_{h} \eta / k T
$$

where $\eta$ is the viscosity and $k T$ thermal energy. According to this equation the radius of the hydrated $\alpha$-chymotrypsin sphere is between 25 and $26 \AA$. This value is consistent with the dimensions of the monomer of $\alpha$-chymotrypsin found by $\mathrm{X}$-ray crystallography (Tsukada \& Blow, 1985; Cohen et al., 1981).

The rise curves of the electric dichroism can also be fitted by single exponentials; however, the quality of least-squares fits is clearly improved by admitting a second exponential according to

$$
\Delta I=\Delta I_{\infty}\left(1-\frac{\tau_{1}}{\tau_{2}-\tau_{1}} e^{-t / \tau_{1}}-\frac{\tau_{2}}{\tau_{2}-\tau_{1}} e^{-t / \tau_{2}}\right)
$$

$\Delta I$ and $\Delta I_{\infty}$ are the changes of the light intensity at time $t$ and $\infty$, respectively. This equation describes rise curves with an initial zero slope (Porschke, 1985). Least-squares fits of experimental rise curves demonstrate that both time constants

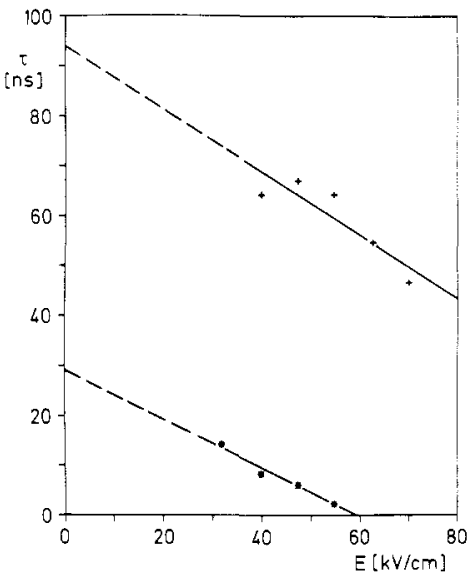

FIGURE 2: Time constants $\tau_{1}\left({ }^{*}\right)$ and $\tau_{2}(+)$ evaluated from dichroism rise curves of $\alpha$-chymotrypsin according to eq 5 as a function of the electric field strength $E$. The straight lines are derived from the experimental data by linear regression (buffer $\mathrm{B} 1,2^{\circ} \mathrm{C}$ ).

$\tau_{1}$ and $\tau_{2}$ decrease with increasing electric field strength. Since the rise curves -in particular those measured at high electric field strengths-are at the limit of time resolution of our technique, the accuracy of the time constants is limited. Within these limits the time constants appear to be a linear function of the electric field strength (cf. Figure 2). A linear regression provides time constants in the limit of zero field strength $\tau_{1}(E=0)=29 \mathrm{~ns}$ and $\tau_{2}(E=0)=94 \mathrm{~ns}$. These values are as expected for the field-induced orientation of permanent dipoles. According to Benoit (1951) $\tau_{1}(E=0)$ should correspond to the time constant of dichroism decay $\tau_{\mathrm{d}}$, whereas the second time constant should be given by $\tau_{2}(E=$ $0)=3 \tau_{\mathrm{d}}$. Thus, the experimental rise curves confirm the conclusion that $\alpha$-chymotrypsin is associated with a permanent dipole moment.

Model Calculations. Bead models for the simulation of hydrodynamic properties of $\alpha$-chymotrypsin were based on known crystal structures (Tsukada \& Blow, 1985; Cohen et al., 1981) as given in the Protein Data Bank. The models are simply formed by substituting one bead for each amino acid. The centers of these beads are located at the centers of mass, which are calculated for any amino acid under the assumption of equal mass for each atom. Since the beads in the resulting models partially overlap, the hydrodynamics can only be simulated for beads of equal radius (Garcia de la Torre \& Bloomfield, 1981). We use a common radius, which is calculated by evaluation of maximal distances of atoms from the 
center of mass for each amino acid, taking the average of these distances $(2.7 \AA)$ and finally adding $2.5 \AA$ to account for hydration. [A bead model representing monomer $\mathrm{A}$ of $\alpha$ chymotrypsin is shown by Antosiewicz and Porschke (1989).] These bead models are then used to calculate rotational diffusion coefficients and centers of diffusion (CD, required for evaluation of dipole moments) according to procedures discussed by Garcia de la Torre and Bloomfield (1981). We have applied a volume correction (Garcia de la Torre \& Rhodes, 1983 ) based on "solvent accessible volumes" developed by Antosiewicz and Porschke (1989).

Dichroism decay curves are calculated for our rigid bead models according to

$$
\xi(t)=\sum_{i=1}^{5} Q_{i} e^{-t / \pi_{i}}
$$

where $\tau_{i}$ are the rotational relaxation times, which depend on rotational diffusion coefficients, and $Q_{i}$ are the corresponding amplitudes, which depend on rotational diffusion coefficients, extinction coefficients, dipole moment, and electric polarizability. Exact expressions have been given by Wegener et al. (1979).

Extinction coefficient tensors at $280 \mathrm{~nm}$ were calculated from the coordinates of tryptophan and tyrosine together with the location of transition moments in these residues. For tryptophan we considered two transitions: one with an extinction coefficient $\epsilon=4800$ (all $\epsilon$ values are given in $\mathrm{M}^{-1} \mathrm{~cm}^{-1}$ ) in the direction defined approximately by the atoms NE1 and CE3 (notation according to the Protein Data Bank) and the second with $\epsilon=800$ in the direction CG and CZ2 (Yamamoto \& Tanaka, 1972; Philips \& Levy, 1986). The contribution of tyrosine (Maki et al., 1978; Fasman, 1976) is described by a transition in the direction CD1-CD2 with $\epsilon=1200$. By tensorial addition of all individual transitions we arrive at a final extinction coefficient tensor, with a total (isotropic) $\epsilon=$ 49600-in agreement with experimental values (Fasman, 1976).

The reduced limiting dichroism $\Delta \epsilon_{\infty} / \epsilon$ was calculated from the extinction coefficients tensor $A$ transformed to the principal axes of the alignment tensor $\chi$ (Wegener et al., 1979; in the present case $\chi$ is a $3 \times 3$ tensor with components $\mu_{i} \mu_{j} / k T^{2}$, where $i$ and $j$ denote two of the coordinates $x, y$, and $z$ )

$$
\frac{\Delta \epsilon_{\infty}}{\epsilon}=3 \frac{\mathrm{A}_{z z}^{*}-\frac{\mathrm{A}_{x x}^{*}+\mathrm{A}^{*}{ }_{v y}}{2}}{\mathrm{~A}^{*}{ }_{x x}+\mathrm{A}^{*}{ }_{y y}+\mathrm{A}_{z z}^{*}}
$$

where $A^{*}{ }_{i i}$ are components of the transformed extinction tensor. The principal axis $z$ of the alignment tensor corresponds to its maximal eigenvalue, which was always 1-2 orders of magnitude larger than any of the two remaining eigenvalues, and thus eq 7 is valid for our evaluations.

Calculations of Dipole Moments. Permanent dipole moments of proteins mainly result from charges on ionized residues and from partial charges on $\alpha$-helices. Thus, the overall dipole moment $\vec{\mu}$ results from two contributions:

$$
\vec{\mu}=\sum q_{k} \vec{r}_{k}+\sum \vec{p}_{l}
$$

The first term simply represents the general definition of the dipole moment, where $q_{k}$ are mean charges of ionized residues and $\vec{r}_{k}$ are their position vectors in a coordinate system with a center, which must correspond to the center of diffusion (cf. above) for particles with a net charge. The second term is calculated as the vectorial sum of the dipole moments $\vec{p}_{l}$ assigned to peptide bonds of $\alpha$-helices. Since $\alpha$-chymotrypsin has two short segments of $\alpha$-helix only (Blow, 1971), their contribution to the total dipole moment is relatively small. We have considered these moments by the assignment of $3.5 \mathrm{D}$ for each $\alpha$-helical peptide bond in the direction from $\mathrm{O}$ to $\mathrm{C}$ of the carbonyl groups (Wada, 1976; Hol, 1985). The dominant part of the dipole moment, which results from the ionized residues, has been calculated by two different procedures.

In the first procedure the mean charges of residues at a given $\mathrm{pH}$ are calculated according to the law of mass action by using $\mathrm{p} K$ values given in the literature (Marini \& Wunsch, 1963; Marini \& Martin, 1971; Fersht \& Sperling, 1973; Cruickshank \& Kaplan, 1972; Ikeda et al., 1982) (3.8 for all carboxyl groups, 7.8 for $\alpha$-amino groups, 10.5 for the $\epsilon$-amino group of lysine, 6.8 for the imidazole $\mathrm{N}$ of histidine, 10.0 for the hydroxyl group of tyrosine, and 12.5 for the guanidinium group of arginine). Because the exact $\mathrm{p} K$ values depend on the local environment of the residues, this procedure provides only approximate dipole moments. However, the uncertainty is limited, because most residues have $\mathrm{p} K$ values far from the $\mathrm{pH}$ values used in our simulations. A special problem results from the fact that 2-3 amino acids at the end of the first polypeptide chain could not be resolved by $X$-ray analysis, and thus the position of the corresponding $\alpha$-carboxyl group has been approximated in our calculation by the coordinates of the last identified atom of this chain. In spite of these limitations the dipole moments calculated by the first procedure can be regarded as reasonable approximations. The main advantage of this procedure is the fact that the evaluation is straightforward and does not require extensive computations.

The second procedure is much more complex. It accounts for electrostatic interactions between sites (Warshel \& Russell, 1984; Rogers, 1986; Warshel et al., 1984; Matthew et al., 1985 ), which is the main source of $\mathrm{p} K$ shifts, and provides information on contributions resulting from charge fluctuations. This information is obtained by generation of an equilibrium distribution of individual states of protein molecules using the Monte Carlo method (Metropolis et al., 1953). The states are protein molecules with their residues in various degrees of protonation. The distribution of states is used to calculate the mean charge of individual residues, the mean dipole moment of the protein, the mean dipolar tensor, and the fluctuating dipole moment defined by

$$
\left(\Delta \mu^{2}\right)^{1 / 2}=\left(\left\langle\sum_{x, y, z} \mu_{i} \mu_{i}\right\rangle-\sum_{x, y, z}\left\langle\mu_{i}\right\rangle^{2}\right)^{1 / 2}
$$

where the angle brackets represent the mean value of the equilibrium distribution.

Individual states of the protein are included in the distribution according to their free energy, which is calculated relative to the protein state without protonation of any site. Any site of the protein $\mathrm{PS}_{k}$ may be protonated to $\mathrm{PS}_{k} \mathrm{H}$. Although this reaction appears to be very simple, its quantitative description is very complex, because several contributions to the free energy have to be considered. We may separate the reaction into several steps. First, the binding site $S_{k}$ may be transferred from the interior of the protein to the aqueous solution (1), which is associated with a particularly large change of the solvation energy if this site bears a charge. Then, the site $S_{k}$ may be protonated (2) corresponding to its standard $\mathrm{p} K_{\mathrm{w}}$ value determined in aqueous solution. In the last step the protonated site $S_{k} H$ is transferred back to the interior of the protein (3); again the change in the solvation energy associated with this step is particularly large if the protonated site is charged:

$\mathrm{PS}_{k}+\mathrm{H}_{\mathrm{w}}^{+} \stackrel{1}{\rightleftharpoons} \mathrm{P}+\left(\mathrm{S}_{k}\right)_{\mathrm{w}}+\mathrm{H}_{\mathrm{w}}^{+} \stackrel{2}{\rightleftharpoons} \mathrm{P}+\left(\mathrm{S}_{k} \mathrm{H}\right)_{\mathrm{w}} \stackrel{3}{\rightleftharpoons} \mathrm{PS}_{k} \mathrm{H}$ 


\begin{tabular}{|c|c|c|c|c|c|c|c|c|c|c|c|c|}
\hline & \multicolumn{4}{|c|}{$\gamma$-chymotrypsin at $\mathrm{pH}$} & \multicolumn{4}{|c|}{$\alpha$-chymotrypsin A at $\mathrm{pH}$} & \multicolumn{4}{|c|}{$\alpha$-chymotrypsin B at $\mathrm{pH}$} \\
\hline & 8.3 & 7.0 & 5.7 & 4.2 & 8.3 & 7.0 & 5.7 & 4.2 & 8.3 & 7.0 & 5.7 & 4.2 \\
\hline \multicolumn{13}{|l|}{ static calculations } \\
\hline mean charge $\mathrm{e}^{b}$ & 0.61 & 3.31 & 5.02 & 9.83 & 0.61 & 3.31 & 5.02 & 9.83 & 0.61 & 3.31 & 5.02 & 9.83 \\
\hline dipole moment ${ }^{c}$ & 2.00 & 1.83 & 1.77 & 1.51 & 1.76 & 1.55 & 1.51 & 1.26 & 1.79 & 1.56 & 1.51 & 1.22 \\
\hline red. limit. dichroism & 0.59 & 0.58 & 0.57 & 0.50 & 0.22 & 0.23 & 0.25 & 0.27 & 0.20 & 0.22 & 0.24 & 0.27 \\
\hline dichroism decay time ${ }^{d}$ & 30.3 & 30.3 & 30.3 & 30.4 & 30.5 & 30.7 & 30.9 & 31.4 & 29.8 & 30.0 & 30.4 & 30.7 \\
\hline \multicolumn{13}{|l|}{ Monte Carlo calculations } \\
\hline mean charge ${ }^{b}$ & 0.79 & 2.75 & 4.76 & 11.7 & 1.65 & 2.65 & 5.10 & 12.1 & 1.77 & 2.52 & 4.87 & 12.0 \\
\hline dipole moment ${ }^{c, e}$ & 1.82 & 1.77 & 1.51 & 0.74 & 1.60 & 1.54 & 1.25 & 0.76 & 1.63 & 1.56 & 1.26 & 0.62 \\
\hline dip. mom. fluct. ${ }^{c f}$ & 0.33 & 0.24 & 0.30 & 0.38 & 0.28 & 0.26 & 0.31 & 0.43 & 0.30 & 0.27 & 0.31 & 0.40 \\
\hline red. limit. dichroism & 0.58 & 0.59 & 0.56 & 0.11 & 0.22 & 0.24 & 0.26 & 0.06 & 0.20 & 0.21 & 0.24 & 0.21 \\
\hline dichroism decay time ${ }^{d}$ & 30.3 & 30.3 & 30.3 & 31.6 & 30.6 & 30.7 & 31.1 & 41.9 & 29.8 & 30.0 & 30.4 & 33.3 \\
\hline translational diffusion coefficients 8 & 9.32 & & & & 9.24 & & & & 9.24 & & & \\
\hline
\end{tabular}

${ }^{a}$ Electrooptical and translation diffusion data refer to $20^{\circ} \mathrm{C}$; rotational relaxation time constants refer to $2{ }^{\circ} \mathrm{C}$. Ionic strength is $0.01 \mathrm{M}$. ${ }^{b} \mathrm{Total}$ charge of the protein in units of elementary charge $e$. ${ }^{c}$ In units of $10^{-27} \mathrm{C} \mathrm{m}$. ${ }^{d} \mathrm{In}$ units of ns. ${ }^{e}$ Root of the largest principal value of the mean dipolar tensor. ${ }^{\prime}$ For definition cf. text. 8 In units of $10^{-7} \mathrm{~cm}^{2} / \mathrm{s}$.

The subscript $w$ denotes that the respective species is in aqueous environment.

The second step of this sequence can be described quantitatively without problem: the "site binding" free energy of a protein state relative to the unprotonated reference state is given by the sum of contributions from individual sites

$$
\Delta G_{\mathrm{s}}=\sum_{k=1}^{m} 2.303 x_{k} k_{\mathrm{b}} T\left(\mathrm{pH}-\mathrm{p} K_{k, w}\right)
$$

where $m$ is the total number of sites; $x_{k}$ is an occupation variable, which is 0 for empty and 1 for occupied sites; $k_{\mathrm{b}}$ is the Boltzmann constant; and $T$ is the absolute temperature.

The other contributions to the free energy resulting from differences in the solvation and from electrostatic interactions between charged residues in the protein cannot be given as readily. We use the approach described by Tanford and Kirkwood (1957) with modifications suggested by Warshel and co-workers (1984). According to these authors the electrostatic free energy of any protein state relative to the unprotonated state is given by

$$
\begin{gathered}
\Delta G_{e}=\frac{e^{2}}{2 b} \sum_{k=1}^{m} \sum_{k \neq l=1}^{m}\left(\xi_{k} \xi_{l}-\gamma_{k} \gamma_{l}\right)\left(A_{k l}-B_{k l}\right)- \\
\frac{e^{2}}{2 a} \sum_{k=1}^{m} \sum_{l=1}^{m}\left(\xi_{k} \xi_{l}-\gamma_{k} \gamma_{l}\right) C_{k l}+ \\
\sum_{k=1}^{m} x_{k}\left(\xi_{k}+\gamma_{k}\right) \frac{e^{2}}{2 \sigma_{k}}\left(\frac{1}{\epsilon_{\mathrm{i}}}-\frac{1}{\epsilon_{\mathrm{w}}}\right)-\sum_{k=1}^{m} x_{k} \frac{e^{2}\left(\xi_{k}^{2}-\gamma_{k}^{2}\right)}{2 b} B_{k k}
\end{gathered}
$$

$b$ is the radius of the protein sphere, which is calculated as the largest distance found between the geometric center of the protein and any atom of the protein-the geometric center is defined as the center of mass and is evaluated with the approximation of equivalent masses for all atoms; $a$ is the radial distance, to which counterions are excluded; $\xi_{k} e$ is the charge of the $k$ th protonated site and refers to states $\mathrm{PS}_{k} \mathrm{H} ; \gamma_{k} e$ is the charge of the $k$ th deprotonated site and refers to states $\mathrm{PS}_{\mathrm{k}} ; \sigma_{k}$ is the radius of the $k$ th ion, which is transferred during reactions (11.1); $\epsilon_{\mathrm{i}}$ and $\epsilon_{\mathrm{w}}$ are the dielectric constants of the protein and of water, respectively; the factors involving $A_{k l}$ represent the energy required for charging residues in an unbounded medium with a dielectric constant of the protein; the factors involving $B_{k l}$ represent the modification due to the fact that the protein is a bounded cavity immersed in a medium of different dielectric constant; the factors involving $C_{k l}$ represent the interaction with counterions in the solvent and thus vanish at zero ionic strength of the solvent. Explicit equations

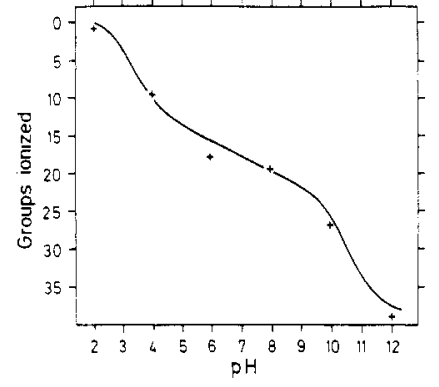

FIGURE 3: Titration curve of $\alpha$-chymotrypsin measured at $20^{\circ} \mathrm{C}$ in $0.15 \mathrm{M} \mathrm{KCl}$ [continuous line, from Marini and Martin (1971)] and data calculated according to the modified Tanford-Kirkwood procedure (+) for the crystal structure of monomer A with $\epsilon_{i}=50, a$ $=b+1.4 \AA$, and $\sigma_{k}=2 \AA$. The calculated data are the mean values of three simulations with 3000 Monte Carlo steps each; standard deviations do not exceed $0.2 x$ (ionizable groups).

for the coefficients $A, B$, and $C$ as well as a more detailed discussion were given by Tanford and Kirkwood (1957). Parts of our results were checked by comparison with published data (Tanford \& Kirkwood, 1957; Orttung, 1968).

By combination of the site binding and the electrostatic plus solvation energy term we get the free energy of a given protein state relative to the unprotonated state

$$
\Delta G=\Delta G_{\mathrm{s}}+\Delta G_{\mathrm{e}}
$$

which is used to assemble the distribution of states according to Monte Carlo procedures. For numerical calculations we use the following parameters: $\sigma_{k}$ is approximated by the van der Waals radius of $\mathrm{NH}_{4}^{+}$and $\mathrm{COO}^{-}$ions as $2 \AA$; the radial "exclusion" distance $a$ is the sum of the protein radius $b$ (cf. above) and a typical ion radius of $1.4 \AA$. The results are strongly dependent upon the choice of the protein dielectric constant. Our value $\epsilon_{\mathrm{i}}=50$, which is much larger than the values assumed by, e.g., Tanford and Kirkwood (1957), Tanford (1961), or Orttung (1968), has been fitted to an experimental protolytic titration curve reported by Marini and Martin (1971) (cf. Figure 3). Obviously, this value should be regarded as an operative parameter and not as a true internal dielectric constant of the protein. Due to the rather high dielectric constant the numerical calculation of the $B$ coefficients require special procedures: the coefficients $B_{k k}$ are calculated according to eq 7 of Tanford and Kirkwood (1957) including 50 Legendre polynomials, whereas the coefficients $B_{k l}$ are calculated in analogy to eq 20 of Tanford and Kirkwood (1957) with development up to $\left(\epsilon_{\mathrm{i}} / \epsilon_{\mathrm{w}}\right)^{4}$ and inclusion of the 10 first Legendre polynominals. 
The final results of the calculations are compiled in Table II. The static dipole moments obtained by both procedures are in satisfactory agreement with the experimental results. The experiments demonstrate a decrease of the dipole moment with decreasing $\mathrm{pH}$. This dependence is correctly represented by both theoretical models. However, the Monte Carlo calculations predict a particularly steep decrease of the dipole moment at $\mathrm{pH}$ 4.2, which is not found experimentally. Apparently, there is some problem associated with the detailed model used for the Monte Carlo calculations in this $\mathrm{pH}$ range. This problem also seems to be the reason for the unusually large relaxation time constant predicted for $\alpha$-chymotrypsin $A$ at 4.2. It should be emphasized that this large time constant does not result from any peculiar value of the individual diffusion coefficients, but is due to a special combination of parameters given in the alignment tensor. The alignment tensor is used to calculate the amplitudes of the five individual relaxation processes, which constitute the dichroism decay curves. Depending upon these amplitudes the apparent time constant fitted to this decay curve may show an acceleration or a retardation effect. The Monte Carlo results for $\alpha$-chymotrypsin at $\mathrm{pH} 4.2$ provide an example for a strong retardation effect. All the other simulated time constants are in very satisfactory agreement with the experimental results.

Although some problem is associated with the Monte Carlo model in the $\mathrm{pH}$ range around 4.2 , this model predicts the dipole moments correctly in the $\mathrm{pH}$ range from 8.3 to 5.7 , and thus the fluctuation moment predicted in this range may be regarded as reliable. As shown in Table II, the fluctuation moments are relatively small compared to the static dipole moments.

Finally, we have also calculated the limit values of the reduced dichroism corresponding to complete molecular alignment. These values are rather sensitive to the location of the transition dipole moments and to the parameters introduced in the alignment tensor. Due to some uncertainty of these values, a perfect agreement of predicted data with experimental results should not be expected. Nevertheless, a comparison reveals that the structure of $\alpha$-chymotrypsin in solution can hardly be identical with that found in crystals for $\gamma$-chymotrypsin. The values predicted for $\alpha$-chymotrypsin A and $B$ are closer to the experimental results, but the agreement is not yet satisfactory - particularly at high $\mathrm{pH}$ values. It remains to be established whether these deviations are due to some problem associated with our model calculations or to a difference between solution and crystal structures.

\section{Discussion}

Permanent dipole moments determined for biological macromolecules did not received much attention-mainly because their nature remained obscure in most cases. Part of the problem comes from the fact that the dielectric method, which has been commonly used for the evaluation of dipole moments, provides the sum of squared moments without much potential for the characterization of the dipole mechanism. Furthermore, the evaluation of dipole moments from dielectric measurements requires a factor of not exactly known numerical value (Takashima, 1972).

The magnitude of macromolecular dipoles and their nature can be characterized in more detail by measurements of the electric dichroism. Stationary values of the dichroism measured as a function of the electric field strength can be used directly to distinguish between induced and permanent dipoles. The numerical values of either the permanent moment or the polarizability can be determined by least-squares fitting at high accuracy. The nature of the dipole moment can be examined independently by a quantitative analysis of the transient dichroism. According to the classical work of Benoit (1951), the dichroism rise curves of permanent dipoles are clearly different from those of induced dipoles. As shown recently for the case of DNA double helices (Porschke, 1985), the special case of a slow polarization mechanism due to "chemical" relaxation may also be characterized quantitatively. Of course, the analysis by dichroism measurements requires that the molecules under investigation are associated not only with an electric but also with an optical anisotropy. Furthermore, the electric dipole should be relatively large for the induction of a sufficiently large degree of orientation at the accessible electric field strengths. Finally, a quantitative analysis of dichroism rise curves requires that the rotational diffusion is not too fast. Recently, this limit has been pushed sufficiently far by application of fast pulse generators and efficient deconvolution techniques, such that most biological macromolecules of interest can be analyzed at a high degree of accuracy.

The dichroism measurements clearly show that our test object $\alpha$-chymotrypsin is associated with a considerable permanent dipole moment. The results of our theoretical calculations confirm the experimental results quantitatively. The permanent dipole is due to an asymmetry of the equilibrium charge distribution, and only a minor contribution comes from fluctuations of the charge distribution. Thus, experiments and theory are in satisfactory agreement with respect to the electric parameters. Nevertheless, we have to comment that the theory of electrostatic interactions deserves further development. This may be illustrated by the fact that the results of the "simple static" model can be regarded as accurate as those obtained from the rather complex Monte Carlo simulation. In the first case, some uncertainty results from not exactly known local $\mathrm{p} K$ values, and in the second case the attempt to calculate local charge states suffers from not precisely known dielectric constants, for example. While the accuracy of predictions according to the simple static model may be improved by exact assignments of local $\mathrm{p} K$ values by NMR measurements, the local dielectric constants required for the more detailed computations cannot be obtained as easily. Since electrostatic interactions contribute a major part to the free energy of proteins, exact knowledge of these interactions is also required for $a b$ initio predictions of protein structures.

To the best of our knowledge the present investigation provides the most detailed comparison of experimental and theoretical results on a protein dipole. We should mention, however, two previous investigations of the corresponding problem. Schlecht (1969) concluded from dielectric measurements and from a simple static dipole calculation that myoglobin is associated with a permanent dipole moment of $170 \mathrm{D}$ (corresponding to $5.7 \times 10^{-28} \mathrm{C} \mathrm{m}$ ). In a series of papers Orttung $(1968,1970)$ has analyzed the nature of the hemoglobin dipole moment and presented data on the $\mathrm{pH}$ dependence of the static and the fluctuating dipole moment. According to this analysis the contribution of the fluctuation dipole moment is larger than that of the static dipole moment at neutral $\mathrm{pH}$. Apparently, general conclusions on the nature of protein dipoles are hardly possible, except that each case should be analyzed separately.

In addition to the electric parameters of the protein, our approach also provides detailed information on the hydrodynamics and the optical anisotropy. Because rotational diffusion is very sensitive to particle dimensions and the optical anisotropy is strongly dependent on the location of the chromophores with respect to the dipole vector, these parameters 
can be used to identify structures in solution. Thus, a combination of electrooptical measurements with detailed model calculations should prove to be very useful for the future analysis of protein structures in solution.

Registry No. $\alpha$-Chymotrypsin, 9004-07-3.

\section{REFERENCES}

Antosiewicz, J., \& Porschke, D. (1989) J. Phys. Chem. 93, 5301-5305.

Benoit, H. (1951) Ann. Phys. 6, 561-609.

Blow, D. M. (1971) Enzymes (3rd Ed.) 3, 185-212.

Bottcher, C. J. F. (1973) Theory of electric polarization, Vol. I, Elsevier, Amsterdam.

Cantor, C. R., \& Schimmel, P. R. (1980) Biophysical Chemistry, Freeman, San Francisco.

Cohen, G. H., Silverton, E. W., \& Davies, D. A. (1981) J. Mol. Biol. 148, 449-479.

Cruickshank, W. H., \& Kaplan, H. (1972) Biochem. J. 130, 1125-1131.

Diekmann, S., Hillen, W., Jung, M., Wells, R. D., \& Porschke, D. (1982) Biophys. Chem. 15, 157-167.

Fasman, G. D., Ed. (1976) CRC Handbook of Biochemistry and Molecular Biology, 3rd ed., Vol. I, p 187, Vol. II, p 406, CRC Press, Cleveland, OH.

Fersht, A. L., \& Sperling, J. (1973) J. Mol. Biol. 74, 137-149.

Fredericq, E., \& Houssier, C. (1973) Electric Dichroism and Electric Birefringence, Clarendon, Oxford, U.K.

Garcia de la Torre, J., \& Bloomfield, V. A. (1981) Q. Rev. Biophys. 14, 81-139.

Garcia de la Torre, J., \& Rhodes, V. (1983) J. Chem. Phys. 79, 2454-2460.

Grünhagen, H. H. (1974) Ph.D. Thesis, Universität Braunschweig.

Hol, W. G. J. (1985) Prog. Biophys. Mol. Biol. 45, 149-195.

Ikeda, K., Kunugi, S., \& Ise, N. (1982) J. Biochem. 92, 541-546.

Kirkwood, J. G., \& Shumaker, J. B. (1952) Proc. Natl. Acad. Sci. U.S.A. 38, 855-862.

Maki, I., Kitaura, K., \& Nishimoto, K. (1978) Bull. Chem. Soc. Jpn. 51, 401-406.
Marini, M. A., \& Wunsch, C. (1963) Biochemistry 2, 1454-1460.

Marini, M. A., \& Martin, C. J. (1971) Eur. J. Biochem. 19, 162-168.

Matthew, J. B., Gurd, F. R. N., Garcia-Moreno, B. E., Flanagan, M. A., Martch, K. L., \& Shire, S. J. (1985) CRC Crit. Rev. Biochem. 18, 91-197.

Metropolis, N., Rosenbluth, A. W., Rosenbluth, M. N., Teller, A. H., \& Teller, E. (1953) J. Chem. Phys. 21, 1087-1092.

Orttung, W. H. (1968) J. Phys. Chem. 72, 4066-4071.

Orttung, W. H. (1970) Biochemistry 9, 2394-2402.

Philips, L. A., \& Levy, D. H. (1986) J. Chem. Phys. 85, 1327-1332.

Porschke, D. (1980) Nucleic Acids Res. 8, 1591-1612.

Porschke, D. (1985) Biophys. Chem. 22, 237-247.

Porschke, D. (1987) Biophys. Chem. 28, 137-147.

Porschke, D., \& Jung, M. (1985) J. Biomol. Struct. Dyn. 2, 1173-1184.

Porschke, D., Meier, H. J., \& Ronnenberg, J. (1984) Biophys. Chem. 20, 225-235.

Porschke, D., Tovar, K., \& Antosiewicz, J. (1988) Biochemistry 27, 4647-4679.

Rogers, N. K. (1986) Prog. Biophys. Mol. Biol. 48, 37-66.

Schlecht, P. (1969) Biopolymers 8, 757-765.

Takashima, S. (1972) Methods Enzymol. 26, 337-362.

Tanford, C. (1961) Physical Chemistry of Macromolecules, pp 477-481, Wiley, New York.

Tanford, C., \& Kirkwood, J. G. (1957) J. Am. Chem. Soc. 79, 5333-5339.

Tsukada, H., \& Blow, D. M. (1985) J. Mol. Biol. 184, 703-711.

Wada, A. (1976) Adv. Biophys. 9, 1-63.

Warshel, A., \& Russell, S. T. (1984) Q. Rev. Biophys. 17, $283-422$.

Warshel, A., Russell, S. T., \& Churg, A. K. (1984) Proc. Natl. Acad. Sci. U.S.A. 81, 4785-4789.

Wegener, W. A., Dowben, R. M., \& Koester, V. J. (1979) J. Chem. Phys. 70, 622-632.

Yamamoto, Y., \& Tanaka, J. (1972) Bull. Chem. Soc. Jpn. $45,1362-1366$. 